\title{
Thread/Cord-Based Microfluidic Devices in Modern Analysis
}

\author{
Deepak Shah and Rohit Bhatia* \\ Department of Pharmaceutical Analysis, ISF College of Pharmacy, India \\ *Corresponding author: Rohit Bhatia, Department of Pharmaceutical Chemistry \& Analysis, ISF College of Pharmacy, \\ India
}

\section{ARTICLE INFO}

Received: 幽 May 10, 2021

Published: 慧 May 25, 2021

\section{ABSTRACT}

Citation: Deepak Shah, Rohit Bhatia. Thread/Cord-Based Microfluidic Devices in Modern Analysis. Biomed J Sci \& Tech Res 36(1)-2021. BJSTR. MS.ID.005795.

\section{Letter}

New ideas and applications of low-price and portable diagnostic technologies have become an attractive area of research interest [1]. The potential of the following technologies in maintaining affordable healthcare and environmental monitoring to developing and remote regions very strongly drives the development of these technologies [2-4]. For the fabrication of low-price and low-volume microfluidic diagnostic devices for healthcare and environmental assays, cotton thread acts as an attractive material for their fabrication because of the gaps in between fibers which provide capillary channels for liquids to wick along the thread. The thoughts of using electronic threads for human health monitoring has been reported in various studies [5-8]. From pioneering works, resorting to expensive and complex manufacturing processes, adopting microfluidic platforms fabricated on polydimethylsiloxane (PDMS) or glass, the research has approached the use of various new types of low price materials and facile manufacturing approaches [913]. The result of this effort is now the development of low price paper-based analytical devices (mPADs) in which fluidic pathways are easily developed by utilizing readily available office tools such as plotters and commercial printers, these last developed with permanent markers that contain hydrophobic inks [14-19].

To decrease the number of steps required for the easy development of low-cost fluidic devices, the utilization of cotton thread has great promise. It is an easily available cellulosic substance with qualities similar to paper, allowing liquid flow by capillarity without any external pumps or forces. Cotton threads are light, hydrophilic, and flexible and do not require any patterning of hydrophobic barriers to restrict liquids within channels. Also, cotton never becomes brittle when wetted by liquids, which is of a different quality from paper [20-24]. Thread has various advantages that make it appropriate to be useful in microfluidicsbased technologies which include its low price, its lightweight, its easy availability, and its hydrophilic nature. The capillary channels present in threads helps in the fluidic flow and it helps to avoid the use of any external pumping system for the flow of fluid [25]. The fine capillary channels of threads efficiently transport liquid which allows thread-based devices to perform semi-quantitative analysis with very low volumes of sample. A huge number of analyses can be performed cheaply and rapidly involving the determination of the analyte's concentration. In a case where a huge number of samples are to be analyzed these methods are considered very valuable for such tasks as disease screening in all the developing countries.

Since thread offers more choices of materials over paper hence thread-based microfluidic sensor has been considered more advantageous over paper-based microfluidic sensors. Since the preparation of paper-based microfluidics is expensive therefore thread is a valuable alternative substance to paper for producing low-cost microfluidics sensors [26]. Cotton threads can become a highly useful candidate for biosensor substrate as it requires very low sample volume and also it has a surface modifiable property for fast reaction time and high sensitivity [27]. From recent studies, it is found that multifilament threads are an inexpensive alternative for the production of low-cost microfluidic devices. These low-cost microfluidic devices can be easily produced by unskilled persons in a very short interval of time and at a very low cost by using 
simple tools, but these devices have shown high potential to be used as medical sensors [28]. Monitoring of some vital biological signals including body temperature, heart rate, blood pressure, and biopotentials which are from the surface of the skin, overall health can be assessed [29]. Non-invasive or blood-free diagnosis can reduce the pain and various risk factors compared with the traditional invasive diagnosis so it's gaining more attention for health status monitoring.

Figure 1 shows the three possible designs of diagnostic devices which are based on the use of cotton thread in which all the three designs wick the fluid from sample application zones by capillary action and reaches the detection zones. The sample application zone is that part where the user deposits the sample whereas the detection zone is that part where the user reads the results of the assay. The first two designs i.e., the woven array device and the branching device depend on similar strategies for loading reagents, defining detection zones, and encapsulating microchannels. The third design which is the sewn array design has the advantage of the thread being sewn into various substrates. In developing countries, for effective control of diseases by a rapid diagnosis of human diseases and detection of environmental contaminants, there is a need for a simple, sensitive, portable analytical device to be built on low-cost materials. These low-cost microfluidic devices can be produced by unskilled persons in a short interval of time and at a low cost by using simple tools. Thread is a suitable material for fabricating microfluidic diagnostic devices for monitoring human health, environment, and food safety, especially for the population in less industrialized areas or remote regions.

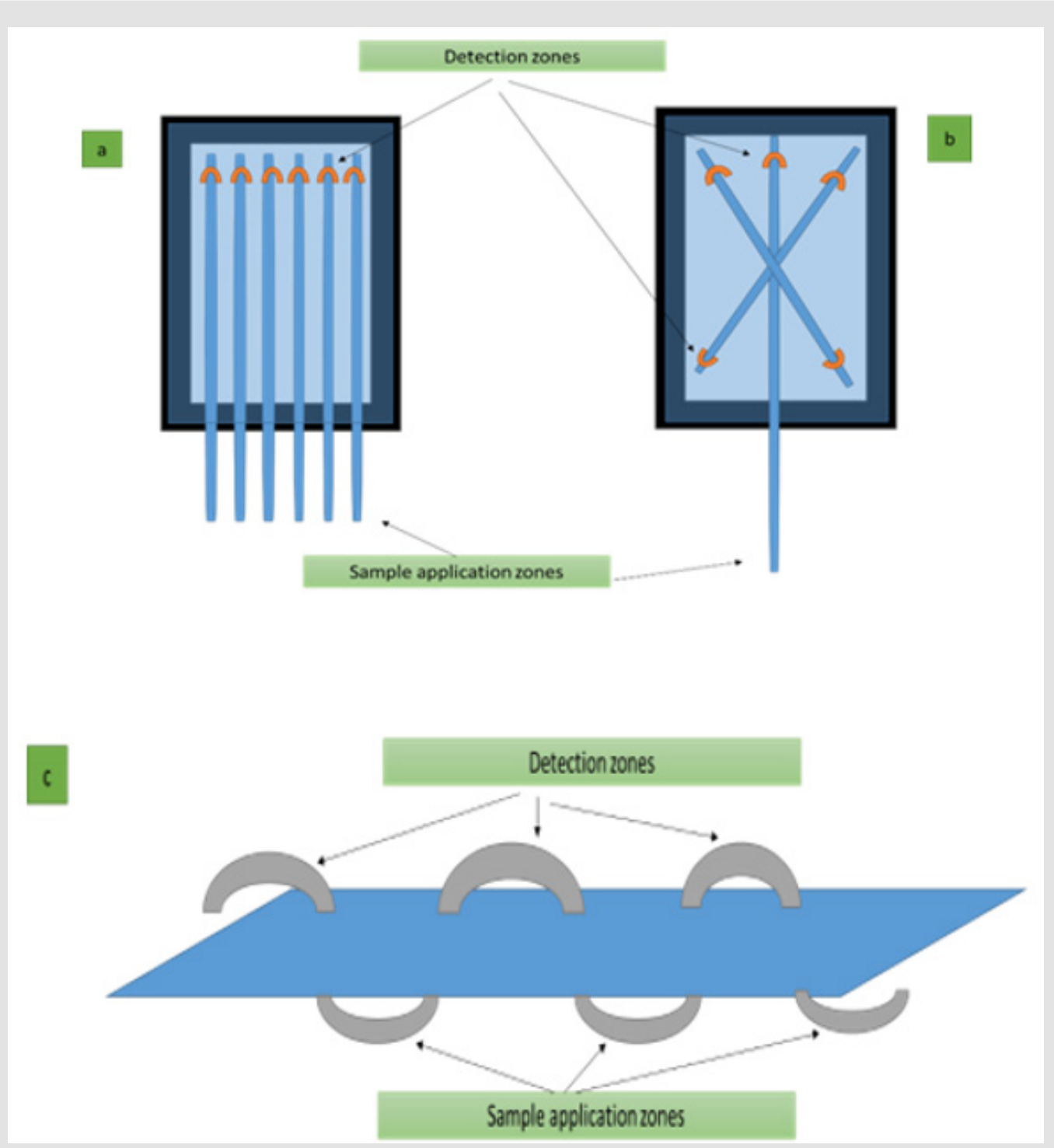

Figure 1: Schematic illustrations of the thread-based devices. Each device comprises detection zones and sample application zones.

(a) The woven array device.

(b) The branching device.

(c) The sewn array design. 


\section{References}

1. Weigl B, Domingo G, LaBarre P, Gerlach J (2008) Towards non- and minimally instrumented, microfluidics-based diagnostic devices. Lab Chip 8(12): 1999-2014.

2. Martinez AW, Phillips ST, Butte MJ, Whitesides GM (2007) Patterned paper as a platform for inexpensive, low-volume, portable bioassays. Angew Chemie Int Ed Engl 46(8): 1318-1320.

3. Abe K, Suzuki K, Citterio D (2008) Inkjet-printed microfluidic multianalyte chemical sensing paper. Anal Chem 80(18): 6928-6934.

4. Leenen FHH, Klement G (1990) Dietary sodium restriction and blood pressure response to sympathetic blockade in young versus adolescent spontaneously hypertensive rats. Can J Physiol Pharmacol 68(1): 46-50.

5. Zhang M, Atkinson KR, Baughman RH (2004) Multifunctional carbon nanotube yarns by downsizing an ancient technology. Science 306(5700): 1358-1361.

6. Shim BS, Chen W, Doty C, Xu C, Kotov NA (2008) Smart electronic yarns and wearable fabrics for human biomonitoring made by carbon nanotube coating with polyelectrolytes. Nano Lett 8(12): 4151-4157.

7. Paradiso R, Loriga G, Taccini N (2005) A wearable health care system based on knitted integrated sensors. IEEE Trans Inf Technol Biomed 9(3): 337-344.

8. Fenton EM, Mascarenas MR, López GP, Sibbett SS (2009) Multiplex lateral-flow test strips fabricated by two-dimensional shaping. ACS Appl Mater Interfaces 1(1): 124-129.

9. Snyder W, Bilbro G, Han YS, Whitaker R, Pizer S (1995) Image Relaxation: Restoration and Feature Extraction. IEEE Trans Pattern Anal Mach Intell 17(6): 620-624.

10. Duffy DC, McDonald JC, Schueller OJA, Whitesides GM (1998) Rapid prototyping of microfluidic systems in poly(dimethylsiloxane). Anal Chem 70(23): 4974-4984.

11. Nath P, Fung D, Kunde YA, Zeytun A, Branch B, et al. (2010) Rapid prototyping of robust and versatile microfluidic components using adhesive transfer tapes. Lab Chip 10(17): 2286-2291.

12. Lucio C, Dominguez H, Neves CA, Brito Neto JGA, Fracassi A (2003) A Dry Process for Production of Microfluidic Devices Based on the Lamination of Laser-Printed Polyester Films. Metabolomics Research 75(15): 3853 3858.

13. Bhattacharjee N, Urrios A, Kang S, Folch A (2016) The upcoming 3D-printing revolution in microfluidics. Lab Chip 16(10): 1720-1742.

14. Coltro WKT, De Jesus DP, Da Silva JAF, Do Lago CL, Carrilho E (2010) Toner and paper-based fabrication techniques for microfluidic applications. Electrophoresis 31(15): 2487-2498.

15. Martinez AW, Phillips ST, Whitesides GM (2010) Diagnostics for the Developing World: microfluidic paper-based analytical devices. Anal Chem 82(1): 3-10.

ISSN: 2574-1241

DOI: $10.26717 /$ BJSTR.2021.36.005795

Rohit Bhatia. Biomed J Sci \& Tech Res

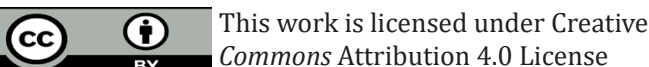

Submission Link: https://biomedres.us/submit-manuscript.php
16. Yamada K, Henares TG, Suzuki K, Citterio D (2015) Paper-based inkjetprinted microfluidic analytical devices. Angew Chemie Int Ed Engl 54(18): 5294-5310.

17. Carrilho E, Martinez AW, Whitesides GM (2009) Understanding wax printing: A simple micropatterning process for paper-based microfluidics. Anal Chem 81(16): 7091-7095.

18. Nie J, Zhang Y, Lin L, Zhou C, Li S, et al. (2012) Low-cost fabrication of paper-based microfluidic devices by one-step plotting. Anal Chem 84(15): 6331-6335.

19. Xia Y, Si J, Li Z (2016) Fabrication techniques for microfluidic paperbased analytical devices and their applications for biological testing: A review. Biosens Bioelectron 77: 774-789.

20. Nightingale AM, Leong L, Burnish RA, Hassan S, Zhang Y, et al. (2019) Monitoring biomolecule concentrations in tissue using a wearable droplet microfluidic-based sensor. Nature Communications 10(3): 224230.

21. Gonzalez A, Estala L, Gaines M, Gomez FA (2016) Mixed thread/paperbased microfluidic chips as a platform for glucose assays. Electrophoresis 37(12): 1685-1690.

22. Mao X, Du TE, Wang Y, Meng L (2015) Disposable dry-reagent cotton thread-based point-of-care diagnosis devices for protein and nucleic acid test. Biosens Bioelectron 65: 390-396.

23. Sateanchok S, Wangkarn S, Saenjum C, Grudpan K (2018) A cost-effective assay for antioxidant using simple cotton thread combining paper based device with mobile phone detection. Talanta 177: 171-175

24. Cabot JM, Breadmore MC, Paull B (2018) Thread based electrofluidic platform for direct metabolite analysis in complex samples. Anal Chim Acta 1000: 283-292.

25. Gaines M, Gonzalez Guerrero MJ, Uchida K, Gomez FA (2018) Microfluidic thread-based electrode system to detect glucose and acetylthiocholine. Electrophoresis 39(24): 3082-3086.

26. Nilghaz A, Ballerini DR, Fang XY, Shen W (2014) Semiquantitative analysis on microfluidic thread-based analytical devices by ruler. Sensors Actuators B: Chem 191: 586-594.

27. Promphet N, Hinestroza JP, Rattanawaleedirojn P, Soatthiyanon N, Siralertmukul K, et al. (2020) Cotton thread-based wearable sensor for non-invasive simultaneous diagnosis of diabetes and kidney failure. Sensors Actuators B: Chem 321: 128549.

28. Ballerini DR, Li X, Shen W (2011) Flow control concepts for threadbased microfluidic devices. Biomicrofluidics 5(1): 1-17.

29. Xiao G, He J, Chen X, Qiao Y, Wang F, Xia Q et al. (2019) A wearable, cotton thread/paper-based microfluidic device coupled with smartphone for sweat glucose sensing. Cellulose 26: 4553-4562.

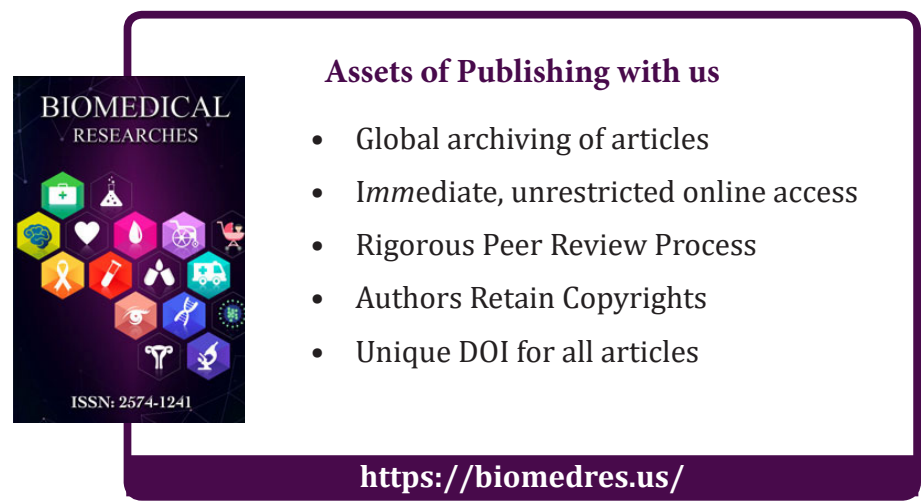

